\title{
然
}

\section{EL MERCADO ENERGÉTICO Y DE RENOVABLES EL CASO DE SIEMENS GAMESA}

El cambio económico que pretende llevar a cabo China hacia un sistema centrado en el consumo y el sector servicios, junto con la desaceleración económica del país, ha propiciado la transformación de su política energética hacia una nueva fase de impulso de la energía limpia, con el objetivo principal de reducir las altas cuotas de contaminación que sufre el país.

Por medio de este artículo, se pretende dar una visión general del cambio energético de China, así como explicar tanto las medidas esenciales tomadas por sus autoridades como la evolución y perspectivas de las principales energías limpias. Finalmente, se abordarán los principales hitos de la empresa Siemens Gamesa, su entrada y sus perspectivas en el sector eólico chino.

Palabras clave: energías renovables, medioambiente, capacidad eólica, certificado de electricidad verde. Clasificación JEL: O53, Q21, Q27, Q58.

\section{El cambio de la política económica y energética en China}

China está decidida a implementar un cambio económico que deje atrás el antiguo modelo en el que se ha sustentado durante años centrado en la industria pesada y las exportaciones, y apostar por una economía orientada al consumo y al sector servicios, impulsando además una industria centrada en la innovación y la alta tecnología'.

\footnotetext{
*Siemens Gamesa Renewable Energy (SGRE) APAC CEO.

${ }^{\star}$ Risk Management Department Siemens Gamesa Renewable Energy (SGRE).

Versión de diciembre de 2017.

1 China National Renewable Energy Centre \& Agora Energiewende. Energy Transition in the Power Sector in China: State of Affairs in 2016; junio de 2017. Recuperado el día 1 de noviembre de 2017. Disponible en: $h$ ttp:// www.agoraenergiewende.de/fileadmin/Projekte/2017/JAW_China_2016/ Agora_Energy-Transition-China-2016-EN_WEB.pdf
}

Las reformas dirigidas al reajuste del crecimiento en favor del consumo y los servicios han hecho que las necesidades energéticas de China cambien al ritmo de la desaceleración económica y se adapten a un crecimiento sostenible del país, dando lugar a que el crecimiento de la demanda de energía disminuya, en comparación con los años de rápido crecimiento económico que experimentó en las pasadas décadas.

La energía desempeña una función clave en dicha transformación económica, de ahí que el país comience una nueva fase de desarrollo liderada por el cambio de la política energética y el impulso de la energía limpia, con el objetivo principal de reducir las altas cuotas de contaminación que sufre el país. 
En este sentido, las autoridades chinas vienen propugnando un cambio de la combinación energética que permita alcanzar los objetivos fijados en los últimos planes quinquenales del Gobierno, algo que pretenden lograr a través del impulso de energías como las renovables o la nuclear en detrimento de los combustibles fósiles, principalmente del carbón.

\subsection{Evolución de la combinación energética en China}

Según datos de la National Energy Administration (en adelante NEA), durante el año 2016 la generación total de electricidad en China alcanzó los 5.989 TWh, un incremento del 5,2 por 100 con respecto al mismo periodo del año anterior $^{2}$, siendo la mayor parte producida por centrales térmicas, principalmente carbón, lo que pone de manifiesto el todavía amplio dominio de este material en la combinación energética del país.

Durante el año 2016 el carbón representó el 62 por 100 de la combinación energética de China, habiendo disminuido dicho porcentaje

2 China Electricity Council. Estadisticas. Disponible en: $h t t p: / / w w w$. cec.org.cn/guihuayutongji/tongjxinxi/niandushuju/2017-01-20/164007.htmI con respecto a 2014 y 2015, cuando representaba el 77 y el 64 por 100 respectivamente. Así, aunque la generación de carbón ha aumentado, su representación porcentual en la combinación energética ha disminuido, principalmente debido al impulso gubernamental de las energías limpias, en línea con los compromisos y objetivos que se han venido fijando.

En 2016, la participación de la energía renovable en la generación de energía ascendió a 1.487 TWh. Durante el primer semestre de 2017, la energía renovable representó el 27,2 por 100 de la generación eléctrica total, aumentando un 1,8 por 100 sobre el mismo periodo del año anterior. Desde 2010, la participación de las energías renovables en la combinación energética ha aumentado un 8 por 100, pasando del 17 por 100 que ocupaba en 2010 al 24,8 por 100 de 2016.

Aunque las energías renovables, especialmente la energía hidroeléctrica, juegan un papel fundamental en la combinación energética de China, la sustitución del carbón por energía renovable, o incluso por energía de bajos niveles de emisión de carbono, como la nuclear o el gas, es aún escasa.

En cualquier caso, la adición de nueva capacidad de energías limpias ha ido $D$

CUADRO 1

ESTADÍSTICAS DE GENERACIÓN DE ENERGÍA ELÉCTRICA EN CHINA EN EL AÑO 2016

\begin{tabular}{|c|c|c|c|}
\hline & 2015 (TWh) & 2016 (TWh) & $\begin{array}{c}\text { Variación } \\
\text { (En porcentaje) }\end{array}$ \\
\hline Generación de electricidad & 5.693 & 5.989 & 5,2 \\
\hline Energía térmica & 4.186 & 4.288 & 2,4 \\
\hline Carbón & 3.853 & 3.905 & 1,3 \\
\hline Gas & 166 & 188 & 12,7 \\
\hline Energía hidroeléctrica & 1.111 & 1.180 & 6,2 \\
\hline Energía nuclear & 171 & 213 & 24,4 \\
\hline Energía eólica & 185 & 241 & 30,1 \\
\hline Energía solar & 38 & 66 & 72,0 \\
\hline
\end{tabular}


aumentando significativamente a lo largo de los últimos años. Así, a finales de 2016, la capacidad instalada de energía hidroeléctrica alcanzó los 332 GW, la capacidad eólica llegó a los $149 \mathrm{GW}$, la energía solar PV a los 77 GW, y la capacidad nuclear a los 33,6 GW. Por su parte, la capacidad instalada de combustible fósil alcanzó los $1.054 \mathrm{GW}$, siendo, con un 5,3 por 100, la energía que menos aumentó en capacidad instalada ${ }^{3}$.

\section{La energía renovable en China}

La gestión de la demanda de energía, así como el control de los niveles de contaminación, suponen un reto para las autoridades chinas, que han fijado como objetivo para 2020 que el 15 por 100 de la energía consumida provenga de fuentes alternativas, como las renovables y la nuclear, disminuyendo así la todavía gran dependencia del carbón.

De esta manera, existe un inexorable cambio del país hacia la energía limpia en línea con su compromiso de ser un gigante económico responsable y concienciado con el medio

3 China Electricity Council, Statistics. Disponible en: $h t t p: / / w w w . c e c$. org.cn/guihuayutongji/tongjxinxi/niandushuju/2017-01-20/164007.html ambiente. Con un creciente consumo eléctrico y una combinación energética todavía dependiente del carbón (aún supone un 65 por 100 de la combinación energética), la necesidad de nuevas fuentes de energía se hace palpable.

China es actualmente el mayor consumidor de energía renovable del mundo, resultado del plan de apoyo e incentivos ofrecidos por el Gobierno chino, y junto a la reducción de costes han hecho que la inversión en el sector se haya incrementado considerablemente durante los últimos años.

Antes del año 2005, la única fuente de energía renovable relevante en China era la hidroeléctrica, que junto con el carbón dominaba la combinación energética del país. A partir de la entrada en vigor de la Ley de Energías Renovables en el año 2006, comenzaron a desarrollarse otras fuentes de energía renovable, como la energía eólica y la solar.

\subsection{Planes del Gobierno chino para el impulso de la energía renovable}

El crecimiento del sector de las energías renovables va ligado a los distintos planes e incentivos puestos en marcha por el Gobierno $\triangleright$

CUADRO 2

CAPACIDAD INSTALADA TOTAL POR FUENTES DE ENERGÍA

\begin{tabular}{|c|c|c|c|}
\hline & 2015 (MW) & 2016 (MW) & $\begin{array}{c}\text { Variación } \\
\text { (En porcentaje) }\end{array}$ \\
\hline Capacidad instalada total & 1.521 .210 & 1.645 .750 & 8,2 \\
\hline Energía térmica & 1.000 .500 & 1.053 .880 & 5,3 \\
\hline Carbón & 895.060 & 942.590 & 5,3 \\
\hline Gas & 66.030 & 70.080 & 6,1 \\
\hline Energía hidroeléctrica & 319.530 & 332.110 & 3,9 \\
\hline Energía nuclear & 27.170 & 33.640 & 23,8 \\
\hline Energía eólica & 131.300 & 148.640 & 13,2 \\
\hline Energía solar & 42.630 & 77.420 & 81,6 \\
\hline
\end{tabular}


chino, que durante los últimos años ha venido destinando una gran cantidad de dinero y recursos con la intención de promover la industria de la tecnología limpia como parte de una política más amplia de independencia energética y protección del medioambiente.

La Ley de Energías Renovables, promulgada en el año 2005, creó las bases y los objetivos necesarios para el impulso de la energía renovable, estableciendo como objetivo diversificar el suministro de energía, salvaguardar la seguridad de la energía, proteger el medioambiente y alcanzar un desarrollo sostenible ${ }^{4}$. Por medio de la modificación de esta misma ley, en diciembre de 2009, se introdujeron además incentivos fiscales y de inversión para promover el sector.

Durante el undécimo Plan Quinquenal del Gobierno chino se presentó el Plan de Desarrollo de Energías Renovables como parte de su política estratégica de energía y de lucha contra el cambio climático, en el cual se establecía un porcentaje del 10 por 100 para 2010 y del 20 por 100 para 2020 en el uso de energías limpias. Asimismo, en su duodécimo Plan Quinquenal (2011-2015), el Gobierno chino estableció como objetivo la reducción del 17 por 100 en la relación carbón/PIB y una participación de energías renovables del 11,4 por 100 para $2015^{5}$.

A principios del año 2017, la NEA presentó el decimotercer plan quinquenal de desarrollo económico y social (2016-2020), que contiene un conjunto de objetivos relacionados en materia energética, entre los que se incluyen un límite de consumo de energía y un objetivo

\footnotetext{
4 China National Renewable Energy Centre. China Renewable Energy Outlook 2016. 1 de noviembre de 2016. Recuperado el 4 de agosto de 2017. Disponible en: http://boostre.cnrec.org.cn/wp-content/ uploads/2016/10/China-Renewable-Energy-Outlook-2016-EN.pdf

5 REN21, Global Status Report 2016. Recuperado el 8 de junio de 2016. Disponible en: http://www.ren21.net/wp-content/uploads/2016/06/ GSR_2016_FullReport_pdf
}

del 15 por 100 en la proporción de recursos no fósiles.

Por otra parte, a principios también del año 2017, el Consejo de Estado Chino aprobó invertir 345.000 millones de euros para proyectos de energías renovables con el objetivo de renovar el modelo de producción energética, impulsar el ahorro de energía y reducir las altas cuotas de contaminación.

Promover la energía renovable es una estrategia a largo plazo de China para responder tanto a la escasez de energía como a la contaminación ambiental. Así, bajo el 13. ${ }^{\text {er }}$ Plan Quinquenal del Gobierno chino se propusieron una serie de objetivos ambiciosos para el incremento de energía renovable: casi duplicar la capacidad eólica terrestre de 128 GW en 2015 a $250 \mathrm{GW}$ en 2020 y triplicar la capacidad de energía solar fotovoltaica de $43 \mathrm{GW}$ en 2015 a 150 GW en 2020.

\subsection{Evolución y perspectivas de la energía solar en China}

En los últimos años, la implementación de energía solar ha cobrado un impulso significativo. En 2015, la capacidad de energía solar fotovoltaica conectada a la red llegó a los $34 \mathrm{GW}$, en 2016 se alcanzaron los 34,5 GW y solo durante los primeros nueve meses de 2017 llegaron a instalarse un total de $43 \mathrm{GW}$ de energía solar6.

En el año 2014, las autoridades chinas fijaron como objetivo para 2020 alcanzar los 100 GW de energía solar instalada; pues $\triangle$

6 Stanford. Steyer-Taylor Center for Energy Policy and Finance. The New Solar System, China's evolving solar industry and its implications for competitive solar power in the United States and the world. Marzo de 2017. Recuperado el 12 de diciembre de 2017. Disponible en: $h t t p s: / / w w w-c d n$. law.stanford.edu/wp-content/uploads/2017/03/2017-03-20-StanfordChina-Report.pdf 
bien, a finales del primer semestre de 2017, la capacidad solar acumulada de China ya había alcanzado los 101,82 GW.

China es, desde el 2013, el principal instalador mundial de energía solar fotovoltaica y también es, desde el 2015, el mayor productor mundial de energía fotovoltaica. Las autoridades chinas han convertido la energía solar en un sector estratégico, lo que les ha llevado a realizar fuertes inversiones en el sector, convirtiendo al país en el mayor inversor de energía solar a nivel mundial, algo que se puso de manifiesto a lo largo del año 2016, cuando el 45 por 100 de las nuevas instalaciones solares del mundo se construyeron en China.

El apoyo gubernamental se basa principalmente en el conocido como feed in tariff, que se estableció en el año 2013 como subsidio para promover el desarrollo tecnológico, la eficiencia energética y la competitividad del sector fotovoltaico. Si bien este sistema de tarifas apoyó el desarrollo de la energía solar en China, también provocó un desmesurado crecimiento por encima del nivel que podía asumir la red, como se vio a lo largo del año 2015, cuando el anuncio de ajuste a la baja de la tarifa hizo que aumentaran considerablemente las instalaciones.

Gran parte de la capacidad se ha instalado lejos de los centros de población, principalmente en las provincias de Xinjiang, principal mercado fotovoltaico de China, seguido de las provincias de Shandong y Henan. A pesar del esfuerzo del Gobierno por fomentar instalaciones distribuidas a pequeña escala, las plantas solares fotovoltaicas a gran escala han continuado representando la mayor capacidad instalada.

Al igual que ha sucedido con la energía eólica, el rápido aumento de la capacidad solar fotovoltaica ha causado problemas de congestión de la red, lo que ha provocado que la inversión comience a dirigirse hacia líneas de transmisión de ultra-alto voltaje para conectar las provincias de producción con las zonas de mayor demanda?

Pese a todo, la energía solar fotovoltaica generó 66,2 TWh de electricidad durante el año 2016, un 69 por 100 más que en el año $\triangleright$

7 China National Renewable Energy Centre. China Renewable Energy Outlook 2016. 1 de noviembre de 2016. Recuperado el 4 de agosto de 2017. Disponible en: http://boostre.cnrec.org.cn/wp-content/uploads/2016/10/ China-Renewable-Energy-Outlook-2016-EN.pdf

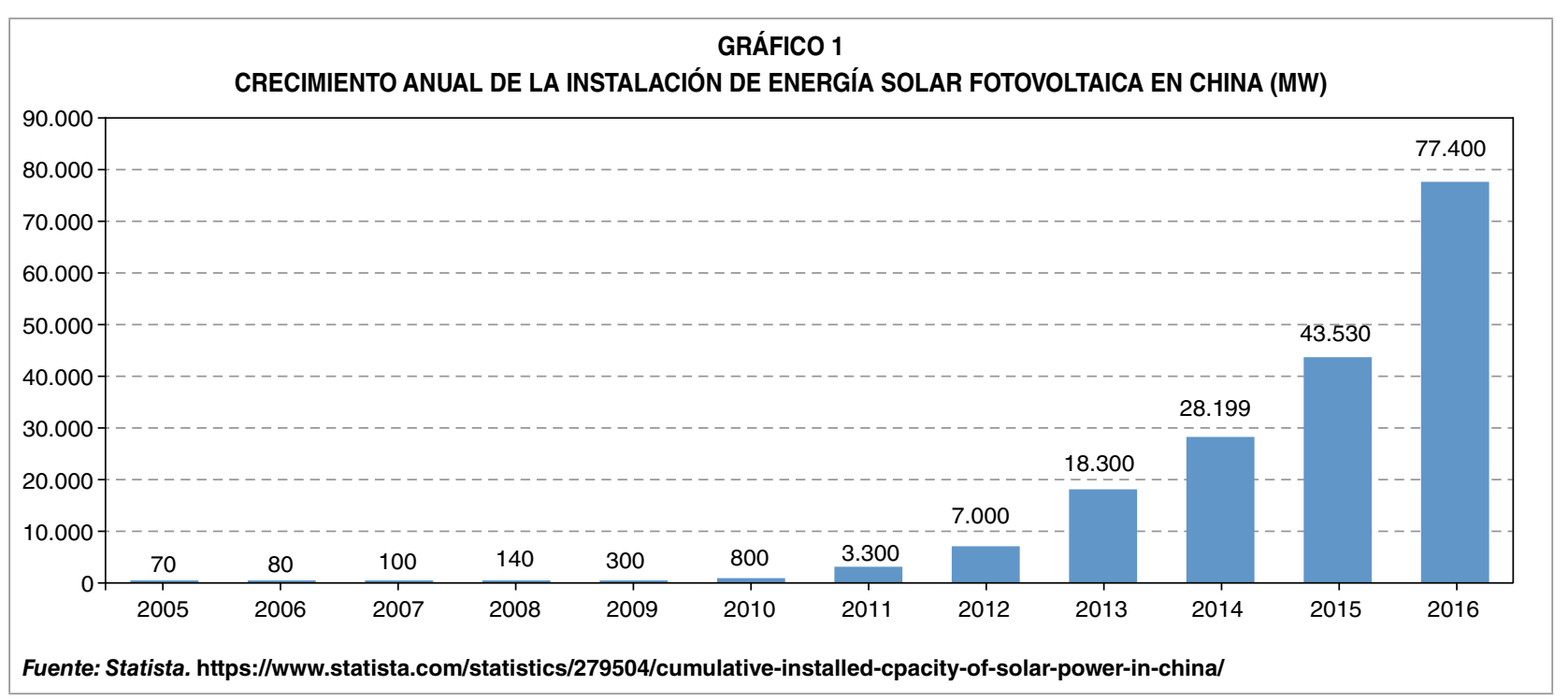


2015, aunque aún continúa representando una cantidad irrisoria, equivalente al 1 por 100, de la combinación energética de China.

\subsection{La energía eólica en China}

De 2005 a 2009, la energía eólica se desarrolló por medio de licitaciones, a través de las cuales se consiguió un gran aumento de la capacidad de energía eólica instalada. A partir del año 2009, el procedimiento de licitación fue reemplazado por el sistema feed in tariff, mediante el cual se fija el precio por kWh producido de acuerdo a la intensidad del viento de la región.

De esta manera, la fijación de precios se divide en cuatro zonas de acuerdo con la intensidad del viento de las diferentes regiones que componen China, estableciendo para cada una de ellas un arancel diferente: cuanto mayor sea la intensidad del viento, menor será el apoyo financiero. Este sistema se estableció con la intención de apoyar el desarrollo de la energía eólica en China e intentar paliar el coste de producción que existía en aquel momento.

China cuenta con abundantes recursos eólicos, lo que ha permitido que experimente un periodo de rápido crecimiento en los últimos diez años, convirtiendo al país en el principal mercado de energía eólica del mundo. Actualmente, China ocupa el primer lugar en capacidad instalada acumulada, con un total de $169 \mathrm{GW}$, representando el 35 por 100 de la suma global.

De los 54,6 GW de capacidad eólica que se instalaron en el mundo en 2016 el 42,7 por 100 correspondían a China, que instaló un total de 23,4 GW, aunque su resultado fue menor que en 2015, cuando se alcanzaron los 30,5 GW. En los próximos diez años se prevé que China instale un promedio anual de más de 25
GW de nueva capacidad eólica, llegando a los $403 \mathrm{GW}$ instalados ${ }^{8}$.

En 2017, la capacidad instalada de energía eólica en China ha continuado creciendo, con un aumento del 13 por 100 en el primer trimestre con respecto al mismo trimestre del año anterior, habiéndose generado un total de 68 TWh de electricidad, un 26 por 100 más que en el mismo trimestre del pasado año ${ }^{9}$.

Los recursos eólicos en China son más abundantes en el norte y noroeste. De esta manera, las provincias del noroeste de China (Región Autónoma de Xinjiang y provincia de Gansu), el noreste de China (Heilongjiang, Jilin, provincias de Liaoning) y el norte de China (provincia de Hebei, Región Autónoma de Mongolia Interior), ocupan más del 90 por 100 del recurso total de energía eólica del Estado, siendo Mongolia Interior la provincia que mayor recurso de energía eólica posee.

Sin embargo, la demanda y el mejor acceso a la red se encuentran principalmente en las regiones costeras. La falta de correspondencia espacial entre los recursos eólicos y la demanda, así como los problemas de integración existentes en la red, se están convirtiendo en las principales limitaciones del desarrollo de la energía eólica en China.

\subsubsection{Los problemas de conexión en la red eléctrica para energía eólica en China}

Pese al aumento anual de la producción, una gran cantidad de energía eólica no $D$

\footnotetext{
8 "China now the world leader in wind power production». The Globe and Mail. Recuperado el 28 de noviembre de 2017. Disponible en: http:// www.theglobeandmail.com/report-on-business/industry-news/energyand-resources/china-now-the-world-leader-in-wind-power-production/ article28713509/

9 Energy Reform Institute NDRC, China National Renewable Energy Centre. China Renewable Energy Outlook 2016. 10 de octubre de 2017. Recuperado el 12 de diciembre de 2017. Disponible en: $h$ ttp://boostre.cnrec. org.cn/wp-content/uploads/2017/11/CREO-2017-booklet-EN-20171109.pdf
} 
CUADRO 3

CAPACIDAD INSTALADA DE ENERGÍA EÓLICA Y PRODUCCIÓN DE ELECTRICIDAD POR PROVINCIAS

\begin{tabular}{|c|c|c|}
\hline Provincia & $\begin{array}{c}\text { Capacidad } \\
\text { instalada } \\
\text { (año 2016, MW) }\end{array}$ & $\begin{array}{c}\text { Producción } \\
\text { de electricidad } \\
\text { (2016, GWh) }\end{array}$ \\
\hline China total & 148,6 & 241,0 \\
\hline Mongolia Interior & 25,5 & 46,4 \\
\hline Xinjiang & 17,7 & 22,0 \\
\hline Gansu & 12,7 & 13,6 \\
\hline Hebei & 11,8 & 21,9 \\
\hline Ningxia & 9,4 & 12,9 \\
\hline Shandong & 8,3 & 14,7 \\
\hline Shanxi & 7,7 & 13,5 \\
\hline Yunnan & 7,3 & 14,8 \\
\hline Liaoning & 6,9 & 12,9 \\
\hline Heilongjiang & 5,6 & 8,8 \\
\hline Jiangsu & 5,6 & 9,8 \\
\hline Jilin & 5,0 & 6,7 \\
\hline Guizhou & 3,6 & 5,5 \\
\hline Guangdong & 2,6 & 5,0 \\
\hline Shaanxi & 2,4 & 2,8 \\
\hline Hunan & 2,1 & 3,9 \\
\hline Fujian & 2,1 & 5,0 \\
\hline Hubei & 2,0 & 3,5 \\
\hline Anhui & 1,7 & 3,4 \\
\hline Sichuan & 1,2 & 2,1 \\
\hline Zhejiang & 1,1 & 2,3 \\
\hline Jiangxi & 1,0 & 1,9 \\
\hline Henan & 1,0 & 1,8 \\
\hline Shanghai & 0,7 & 1,4 \\
\hline Qinghai & 0,6 & 1,0 \\
\hline Guangxi & 0,6 & 1,3 \\
\hline Hainan & 0,3 & 0,6 \\
\hline Tianjin & 0,2 & 0,6 \\
\hline Chongqing & 0,2 & 0,5 \\
\hline Beijing & 0,1 & 0,3 \\
\hline Tibet & 0,1 & 0,1 \\
\hline \multicolumn{3}{|c|}{$\begin{array}{l}\text { Fuente: National Energy Administration, 2016a, } 2015 \text { wind power } \\
\text { installations and production by province. } \\
\text { Disponible en: www.nea.gov.cn/2016-02/04/c_135073627.htm }\end{array}$} \\
\hline
\end{tabular}

puede conectarse a la red eléctrica, lo que da lugar a lo que se conoce como curtailment, es decir, la cantidad total de la energía eléctrica que, pudiendo ser generada por los aerogeneradores de acuerdo con los recursos eólicos, es eliminada debido a los límites existentes en las líneas de transmisión eléctrica o por los posibles problemas de seguridad en la red.

Las razones residen en las imperfecciones existentes en la red y en la falta de correspondencia espacial entre las provincias con mayores recursos eólicos y las provincias o regiones con mayor demanda de energía. De esta manera, la reducción se ha producido, principalmente, en áreas donde el crecimiento de la demanda de energía no ha coincidido con el crecimiento de la oferta.

Este problema ha existido principalmente en las regiones que cuentan con un menor desarrollo de la red y que a su vez poseen mayores recursos eólicos, como las provincias del norte de China (Gansu, Mongolia Interior y Jilin). En estas provincias, el crecimiento del suministro eléctrico no ha seguido el ritmo de la demanda, lo que hace necesaria la exportación o la eliminación de la producción en caso de que la primera no sea posible.

La exportación de energía a los centros de demanda en el sur y en el este del país se encuentra restringida debido a la limitada capacidad de transmisión y a la falta de flexibilidad en los procesos y acuerdos que rigen la transmisión de energía entre las regiones. Por otra parte, la limitada capacidad de transmisión provoca, asimismo, que los operadores de red rechacen la energía eólica por temor a que desestabilice la red y provoque interrupciones operativas.

En consecuencia, los operadores de red continúan despachando energía proveniente de fuentes de generación térmica y eliminando energía eólica, algo que aunque la Ley de Energías Renovables de 2005 y las posteriores enmiendas de 2009 intentaron impedir, obligando a los operadores de red a comprar y despachar toda la energía generada por $\triangle$ 
fuentes de energía renovable, no siempre se cumple ${ }^{10}$.

De esta manera, entre 2010 y 2016, la tasa media de rechazo de energía eólica en China superó el 10 por 100, incrementándose en el primer semestre de 2016, cuando se llegó al 17 por 100 (un 6 por 100 más que la experimentada en el año 2015), echándose a perder 13 TWh de electricidad debido a las imperfecciones existentes en la red ${ }^{11}$.

A fin de paliar los problemas de red, el Gobierno chino ha venido impulsando un programa de inversión en líneas de transmisión de voltaje ultraalto (UHV), que minimiza las pérdidas de transmisión a largas distancias. Estas líneas se consideran como uno de los principales medios para abordar la reducción, ya que facilitan la transmisión de la energía eólica de las provincias ricas en recursos eólicos a los centros de demanda.

\subsection{La carga financiera por subsidios y el nuevo sistema Green Electricity Certificate}

Las energías renovables en China han aumentado de forma exponencial durante los últimos años apoyadas en los subsidios facilitados por el Gobierno chino, lo que ha supuesto que a medida que el crecimiento de la capacidad de energía renovable aumentaba, la carga de inversión estatal creciera también a un nivel muy alto.

La energía proveniente de fuentes eólicas y solares es más cara que la energía a carbón

\footnotetext{
10 "China now the world leader in wind power production", The Globe and Mail. Recuperado el 28 Noviembre de 2017. Disponible en: $h t t p: / /$ www.theglobeandmail.com/report-on-business/industry-news/energyand-resources/china-now-the-world-leader-in-wind-power-production/ article28713509/

11 China National Renewable Energy Centre. China Renewable Energy Outlook 2016. 1 de noviembre de 2016. Recuperado el 4 de agosto de 2017. Disponible en: http://boostre.cnrec.org.cn/wp-content/ uploads/2016/10/China-Renewable-Energy-Outlook-2016-EN.pdf
}

en China, pero el precio pagado por la red es el mismo, independientemente de dónde provenga dicha energía. Para fomentar la inversión en energías renovables, el Estado ha apoyado hasta ahora a las empresas energéticas subvencionando directamente el precio pagado por la red, es decir, el costo adicional de la compra de electricidad renovable en comparación con el carbón.

Pero el sistema de subsidios no está funcionando, la rápida expansión de las energías renovables ha aumentado la carga financiera sobre el Estado y los pagos tardan en llegar, lo que dificulta que las empresas de energía recuperen sus inversiones.

Ello ha supuesto que la Comisión Nacional de Desarrollo y Reforma de China, el Ministerio de Finanzas y la Administración Nacional de Energía anunciaran, en febrero de 2017, un programa piloto para la emisión de certificados verdes a productores de energía renovable. Mediante este nuevo sistema se pretende reducir la carga financiera del Gobierno eliminando los subsidios directos para las energías renovables y trasladar el coste a las compañías.

Esto ayudará a las empresas productoras de electricidad renovable a ver un retorno más rápido de la inversión porque los certificados se adjudicarán a los generadores tan pronto como la energía se entregue a la red.

Estos certificados representarán, cada uno, $1 \mathrm{MW}$ de electricidad producida por generación solar o eólica, y serán los generadores y minoristas de electricidad los que tendrán que adquirir estos certificados de electricidad verde que serán los que demuestren que la electricidad se ha generado a través de fuentes de energía renovables.

Este sistema hará disminuir el monto total de subsidios recibidos por los productores de energía renovable, pero hará que mejore la $D$ 
liquidez reduciendo los retrasos experimentados por el fondo de energía renovable.

\section{La compañía Siemens Gamesa en China}

Siemens Gamesa Renewable Energy se constituyó en abril de 2017 tras la fusión de Gamesa Corporación Tecnológica y Siemens Wind Power, filial eólica de Siemens, creando una de las primeras compañías de la industria eólica, con presencia en más de noventa países y con capacidad industrial en los principales mercados eólicos y una base instalada de $83 \mathrm{GW}$.

Asimismo, esta operación ha reforzado la presencia de la compañía en el mercado chino, al unir el potencial de Siemens en la eólica marina y su consolidada presencia en mercados maduros de Asia-Pacífico, como Australia y Japón, con el know-how y competitividad de los aerogeneradores terrestres de Gamesa y su fortaleza en China, India y los mercados del Pacífico Occidental.

Con una trayectoria de quince años en el mercado chino, cuando Gamesa entró en el país, Siemens Gamesa cuenta con una fuerte presencia en uno de los países del mundo con mayor crecimiento de energía eólica; en Tianjin tiene su mayor base de producción de aerogeneradores fuera de España. Además, en Pekín tiene su sede central para los mercados de Asia-Pacífico, como Vietnam, Indonesia, Filipinas, Taiwán, Corea del Sur, Japón, Pakistán, Sri Lanka o Tailandia.

Entre otros hitos, Siemens Gamesa instaló su primera turbina eólica en China en el año 2000, en el parque eólico de Yumen, localizado en la provincia de Gansu, para la compañía Longyuan Power, el mayor productor de energía eólica de China.
En 2005, la compañía estableció en Tianjin uno de sus centros globales de producción y suministro, donde fabrica nacelles, generadores, convertidores y armarios de control.

En el año 2009 desarrolló su primer parque eólico en China, Taipingshan 1, en la ciudad de Weifang, provincia de Shandong, con 58 turbinas con una potencia total de $49 \mathrm{MW}$. $\mathrm{Y}$ en el año 2012, se convertía en el primer fabricante de aerogeneradores extranjero en China por capacidad instalada.

Desde su centro productivo en Tianjin, exportó en el año 2012 un total de 35 turbinas con destino México; asimismo, ese mismo año, las turbinas eólicas de Siemens Gamesa pasaron la prueba LVRT (Low Voltage Ride Through) en China, que permite que permanezca conectada a la red durante una caída de tensión o un fallo de red, algo crucial en el sistema de control de la turbina eólica en China, al producirse desconexiones de parques eólicos con relativa facilidad.

En 2015, la sólida presencia local y el desarrollo de una competitiva cadena de suministro, con un grado de localización del 89 por 100, llevó a la compañía a vender más de $410 \mathrm{MW}$ en China, un 13 por 100 de las ventas totales de aerogeneradores, y a convertirse en el primer fabricante extranjero por cuota de mercado.

Durante ese mismo año se firmaron dos nuevos contratos para el desarrollo de proyectos complejos en zonas con necesidades especiales. Por una parte, se instalaron 24 turbinas en el parque eólico de Dingyanshan, ubicado en la provincia costera de Fujian, frecuentemente afectada por tifones. Asimismo, se suministraron 24 turbinas para el parque eólico de Cangfang, situado a 3.400 metros de altura.

El desarrollo tecnológico de la compañía se ha convertido en una de sus principales ventajas competitivas, al ofrecer productos específicamente adaptados a las necesidades $\triangleright$ 


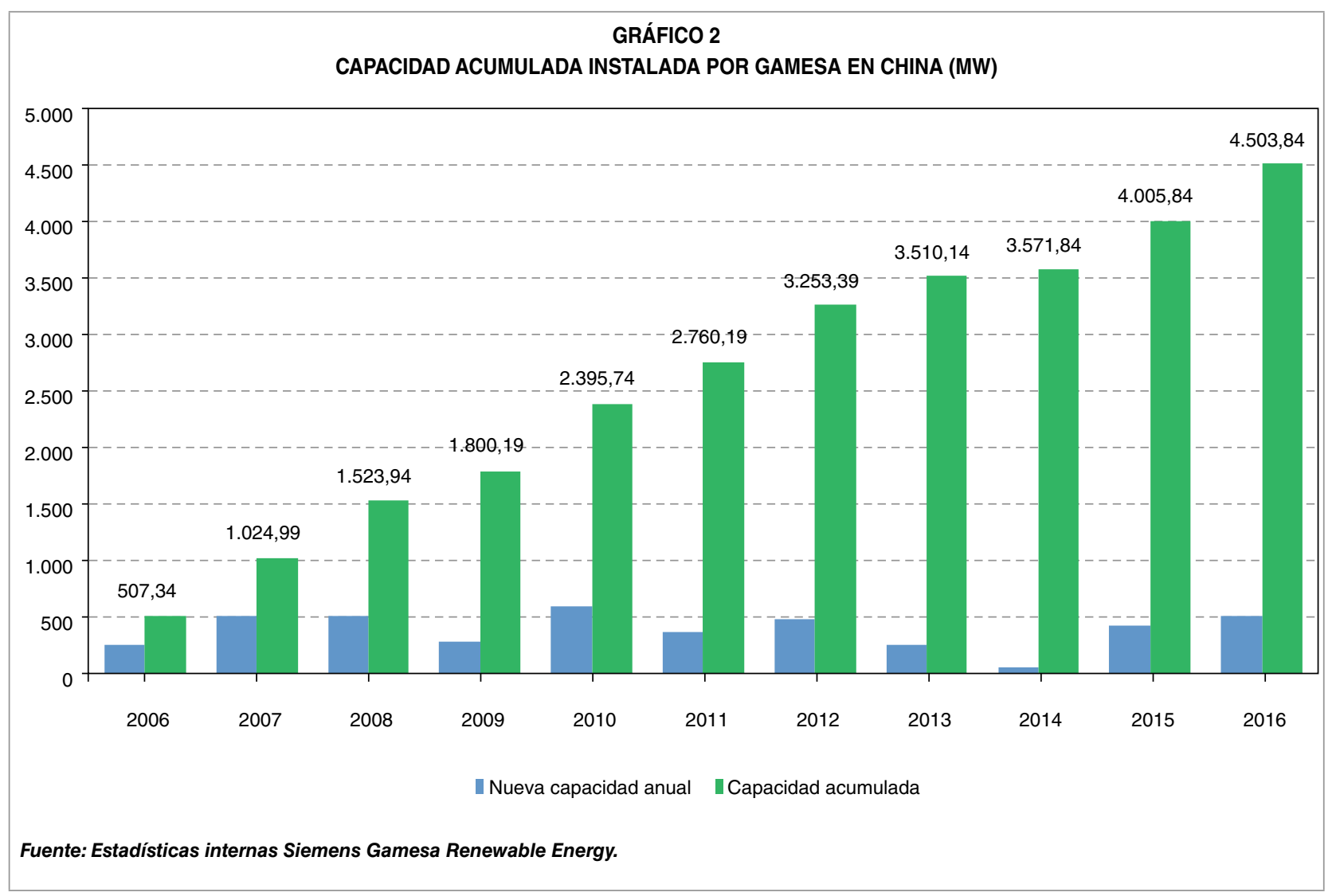

del mercado chino, como turbinas capaces de soportar los fuertes vientos costeros de la provincia de Fujian o las temperaturas extremadamente bajas del noroeste del país.

Por otro lado, cabe destacar que Siemens Gamesa ha obtenido el certificado local de la Administración Nacional de Energía china a la turbina G97-2.0 MW, una máquina de referencia en este mercado con más de 150 unidades instaladas desde 2011. Gamesa se ha convertido en el primer fabricante extranjero que consigue que una agencia de certificación local le otorgue este reconocimiento, requisito obligatorio para poder comercializar turbinas en el país.

En el año 2016, Gamesa ocupó el segundo puesto entre los fabricantes de aerogeneradores extranjeros en China, con un total de 498 MW instalados. Desde 2012, la participación de mercado de los fabricantes de aerogeneradores se ha ido concentrando, hasta reducirse a veinticinco empresas, que compiten por el 5 por 100 de cuota de mercado reservada para los fabricantes extranjeros.

\section{Bibliografía}

[1] BALL, J.; REICHER, D.; SUN, X. y POLLOCK, C. (2017). The New Solar System: China's Evolving Solar Industry and Its Implications for Competitive Solar Power in the United States and the World. Stanford Univ., CA (United States): Steyer-Taylor Center for Energy Policy and Finance. Recuperado el 12 de diciembre de 2017. Disponible en: https://www-cdn.law.stanford.edu/wp-content/uploads/2017/03/2017-0320-Stanford-China-Report.pdf

[2] BLACKWELL, R. (2016, febrero). «China now the world leader in wind power production». The Globe and Mail (en línea). Retrieved 28 February 2016. $\triangleright$ 
Disponible en: http://www.theglobeandmail. com/report-on-business/industry-news/ energy-and-resources/china-now-theworld-leader-in-wind-power-production/ article28713509/

[3] CHINA NATIONAL RENEWABLE ENERGY CENTRE (2016). China Renewable Energy Outlook 2016. 1 de noviembre de 2016. Recuperado el 4 de agosto de 2017. Disponible en: http://boostre.cnrec.org.cn/wp-content/ uploads/2016/10/China-Renewable-EnergyOutlook-2016-EN.pdf

[4] CHINA NATIONAL RENEWABLE ENERGY CENTRE \& AGORA ENERGIEWENDE (2017). Energy Transition in the Power Sector in China: State of Affairs in 2016; junio de 2017. Recuperado el 1 de noviembre de 2017.

[5] ENERGYREFORM INSTITUTE NDRC, CHINA NATIONAL RENEWABLE ENERGY CENTRE (2017). China Renewable Energy Outlook 2016. 10 de octubre de 2017. Recuperado el 12 de diciembre de 2017. Disponible en: $h$ ttp://boostre. cnrec.org.cn/wp-content/uploads/2017/11/ CREO-2017-booklet-EN-20171109.pdf

[6] NATIONAL DEVELOPMENT AND REFORM COMMISSION (2017). Relative Policies on Deepening the Reform of Power Industry. Recuperado el 12 de noviembre de 2017. Disponible en: http://tgs.ndrc.gov.cn/zywj/ 201601/t20160129_773852.html

[7] NATIONAL ENERGY ADMINISTRATION (2016a). 2015 wind power installations and production by province. Recuperado el 17 de noviembre de 2017. Disponible en: www.nea. gov.cn/2016-02/04/c_135073627.htm

[8] NATIONAL ENERGY ADMINISTRATION (2017). Wind Power Integration in the first half of 2016. 26 de julio de 2016. Recuperado el 3 de agosto de 2017. Disponible en: $h t t p: / / w w w$. nea.gov.cn/2016-07/26/c_135540902.htm

[9] REN21 (2016). Global Status Report 2016. Recuperado el 8 de noviembre de 2016. Disponible en: $h t t p: / / w w w . r e n 21 . n e t / w p-c o n t e n t /$ uploads/2016/06/GSR_2016_FullReport_. pdf 
